\title{
Beta-2 microglobulina para detección de enfermedad renal en la asociación del adulto mayor
}

\section{Beta-2 microglobulin for detection of kidney disease in the association of the elderly}

\author{
Alexander Darío Castro Jalca \\ 12alexandercastro@gmail.com \\ Hospital General del Norte Los Ceibos, Guayaquil \\ Ecuador \\ https://orcid.org/0000-0002-5611-8492 \\ Erick Xavier Marcillo Proaño \\ erickmapro@hotmail.com \\ Hospital de Especialidades Portoviejo, Portoviejo \\ Ecuador \\ https://orcid.org/0000-0002-0002-2950 \\ Jazmín Elena Castro Jalca \\ jazmin.castro@unesum.edu.ec \\ Universidad Estatal del Sur de Manabí, Jipijapa \\ Ecuador \\ https://orcid.org/0000-0002-3383-105X \\ Javier Martín Reyes Baque \\ javier.reyes@unesum.edu.ec \\ Universidad Estatal del Sur de Manabí, Jipijapa \\ Ecuador \\ https://orcid.org/0000-0003-0928-1833
}

Recibido: 6 de septiembre de 2019

Aprobado: 28 de septiembre de 2019

\section{RESUMEN}

La enfermedad renal es el resultado final de una serie de situaciones patológicas que causan daño irreversible al riñón, terminando en una pérdida progresiva de la función renal, que generalmente tiene consecuencias letales si el paciente no recibe tratamiento de diálisis o un trasplante. Según la Organización Mundial de la Salud, constituye una causa importante de muerte y discapacidad y estima que su tendencia crecerá de manera exponencial, fundamentalmente entre las poblaciones vulnerables, 
presentándose como una amenaza para la salud pública. Este estudio tuvo por objetivo determinar la prevalencia de la enfermedad renal en los miembros de la Asociación de Ancianos del Cantón Jipijapa, mediante el análisis de indicadores de concentración sanguínea del polipéptido Beta-2 Microglobulina, por métodos turbidimétricos; y relacionarlo con los factores de riesgo asociados al deterioro de la función renal, que se revelaron en las consultas de hábitos realizadas a estas personas mayores, como una acción profiláctica y detección temprana del padecimiento. Se empleó un método descriptivo de corte transversal, basado en un muestreo probabilístico que incluyó 49 individuos con una edad promedio de 71 años, los que se sometieron a los exámenes y encuestas. Los datos fueron recopilados en una ficha de registro y procesados en SPSS para su análisis. A pesar de que el $75.5 \%$ de los sujetos reflejaron valores aceptables en las pruebas de Microglobulina Beta-2, no se puede descartar que el resto presentara resultados considerados superiores a lo normal, corroborando la hipótesis inicial de que más del 15\% de la población de estudio tenía valores elevados de concentración de las moléculas en estudio, lo que indica que desarrollaban una enfermedad renal. Los principales factores de riesgo asociados detectados corresponden, en orden de prioridad, a la hipertensión arterial y a la diabetes mellitus tipo 2. La relación entre la exposición al riesgo y los resultados de la prueba confirmaron su influencia y el efecto nocivo sobre los afectados.

Descriptores: Enfermedad Renal; Adulto Mayor; Factores de Riesgo; Beta-2 Microglobulina.

\begin{abstract}
Renal disease is the final result of a series of pathological situations that cause irreversible damage to the kidney, ending in a progressive loss of renal function, which usually has lethal consequences if the patient does not receive dialysis treatment or a transplant. According to the World Health Organization, it is an important cause of death and disability and it estimates that its tendency will grow exponentially, fundamentally among vulnerable populations, presenting itself as a threat to public health. The objective of this study was to determine the prevalence of kidney disease in the members of the Association of Elders of the Jipijapa Canton, by analyzing blood concentration indicators of the Beta-2 Microglobulin polypeptide, by turbidimetric methods; and to relate it to the risk factors associated with the deterioration of renal function, which were revealed in the consultations of habits performed on these elderly people, as a prophylactic action and early detection of the disease. A descriptive crosssectional method was used, based on a probabilistic sampling that included 49 individuals with an average age of 71 years, who underwent examinations and surveys. The data was collected in a registration form and processed in SPSS for analysis. Despite the fact that $75.5 \%$ of the subjects reflected acceptable values in the Beta-2 Microglobulin tests, it can not be ruled out that the rest presented results considered superior to the normal, corroborating the initial hypothesis that more than $15 \%$ of the population. The study had high concentrations of the molecules under study, which indicates that they developed kidney disease. The main associated risk
\end{abstract}


factors detected correspond, in order of priority, to arterial hypertension and type 2 diabetes mellitus. The relationship between the risk exposure and the results of the test confirmed its influence and the harmful effect on those affected.

Descriptors: Renal Disease; Older Adult; Risk Factors; Beta-2 Microglobulin.

\section{INTRODUCCIÓN}

Según la Organización Mundial de la Salud (OMS), a pesar de que la Enfermedad Renal Crónica constituye una importante causa de muerte y discapacidad a nivel mundial, la sensibilización sobre el tema entre pacientes y distribuidores de salud continúa siendo escasa e insuficiente. Estima además, que esta dolencia crecerá con rapidez entre las poblaciones vulnerables, constituyendo el aumento de su prevalencia, una amenaza para la salud pública global, y recomienda en cuanto a la prevención y control, la mejora en la vigilancia, el tamizaje y la educación de los entes de salud, pacientes y público en general. (Organización Mundial de la Salud (OMS), 2018).

En nuestro país, las estadísticas del Ministerio de Salud (MSP) reportan que el número de decesos por este concepto está aún por debajo del porcentaje que registran las organizaciones internacionales para la región, pero reconoce la preocupación por el incremento exponencial del padecimiento, teniendo en cuenta entre otros factores, que dos de las enfermedades desencadenantes (diabetes mellitus e hipertensión arterial), ocupan los primeros lugares entre las cifras de eventos letales aportadas por el Instituto Nacional de Estadísticas y Censos (INEC) y los registros del propio ministerio, sobre todo cuando los modos y el estilo de vida promedio de la población propician el desarrollo de este tipo de aflicciones.

Un caso especial de atención lo constituye la población considerada como adulto mayor, ya que la frecuencia de la patología se ve incrementada con la edad, producto al detrimento natural de los órganos, constituyendo uno de los factores de riesgo no modificables con mayor incidencia en su progresión. Por ende, en esta etapa de la vida debemos prestar una mayor atención a la profilaxis para mantener parámetros aceptables de salud en los ancianos y con ellos, garantizar su calidad de vida.

El riñón ocupa un lugar relevante en el proceso de envejecimiento y en sus consecuencias sobre la operatividad general del organismo, desempeñando un papel 
fundamental en el mantenimiento del equilibrio metabólico del cuerpo humano. Los hábitos alimentarios, las comorbilidades, el consumo de varios medicamentos y algunas costumbres constituyen una fuente de daño para este órgano, cuya función más importante consiste en la concentración y excreción de metabolitos y drogas tóxicas, convirtiendo a los ancianos en un blanco importante para el compromiso de la función renal, que sumado a otras condiciones como el detrimento miocárdico, la aparición de enfermedades vasculares y una depleción en el volumen sanguíneo, ponen en verdadero riesgo a estos individuos.

En esta disyuntiva se presenta como problema evaluar el comportamiento del daño renal en la población de la Asociación del Adulto Mayor del cantón Jipijapa y su relación con los factores de riesgo más recurrentes entre los ancianos de esta comunidad, con el objetivo de mejorar los sistemas de prevención de salud, adecuándolos a sus condiciones y necesidades específicas, haciendo cada vez más efectiva la acción profiláctica.

La identificación precoz de los pacientes con insuficiencia renal crónica, permite retrasar la progresión de la enfermedad y modificar los factores de riesgo asociados. No obstante, existen pocos estudios que nos muestren la realidad de esta entidad, situación que se ve favorecida debido a que los pacientes en la mayoría de los casos no acuden al especialista sino a un médico de atención primaria, dificultando la obtención real de los datos epidemiológicos. (Fernández Vega, y otros, 2005)

En el caso específico de Jipijapa permitiría, extrapolando los resultados, obtener una valoración del comportamiento de esta enfermedad en el grupo etario de referencia y sus factores de riesgo más comunes, con el objetivo de desarrollar acciones de prevención que repercutan en el mejoramiento y las garantías de este segmento tan sensible.

Para llevar a cabo el estudio, se estableció como objetivo general, determinar la prevalencia del padecimiento de la enfermedad renal en los ancianos de la institución, a partir del análisis en suero sanguíneo del polipéptido Beta-2 Microglobulina, mediante el método de turbidimetría, la identificación de los factores de riesgo en los adultos mayores en la comunidad, y establecer la relación existente entre ambos 
aspectos, con lo cual instituir las bases para capacitarlos sobre la adecuación de sus hábitos y con ello minimizar los efectos del padecimiento.

Ya en desuso, la determinación de las concentraciones de beta2-microglobulina (B2M) se utilizó en la clínica práctica para la estimación del desarrollo de la enfermedad renal, aunque en la actualidad se han encontrado nuevas aplicaciones clínicas para este parámetro, generalmente como marcador de activación inmune en diversas infecciones virales, procesos inflamatorios y más recientemente es muy utilizada en análisis tumorales. (Gazapo E. G., 1996)

El método de elección preferente es la evaluación del filtrado glomerular, cuya tasa de disminución, suele ser una de las anormalidades que comúnmente se presentan tanto en el fallo renal agudo como en el crónico. La medición de la creatinina y otros nitrogenados en plasma, o la depuración de creatinina en la orina, se empleaban como pruebas indirectas para cuantificar la tasa de filtración glomerular, a pesar de que en ocasiones pueden presentar una baja sensibilidad diagnóstica, además de la incomodidad operacional en el caso de la prueba de orina, lo que ha derivado los análisis, al control de Cistanina C, interleucina-18 (IL-18), molécula-1 de lesión renal (kidney injury molecule-1: KIM-1), N-acetil-b-Dglucosaminidasa (NAG), isoforma-3 del intercambiador de sodio-hidrógeno (sodium-hydrogen exchanger isoform-3: NHE-3) y lipocalina asociada con la gelatinasa de neutrófilos (neutrophil gelatinase associated lipocalin: NGAL).

Con estos últimos marcadores se ha resuelto el incremento del umbral de sensibilidad, que representaban los descensos importantes de la TFG, en estadios iniciales de la enfermedad renal, acompañados de cambios leves en la concentración de creatinina, así como los grandes cambios de concentración de esta última con una variación moderada del TFG en las fases avanzadas. Sin contar la influencia en su variación por diversos factores extra-renales.

El criterio de elección del empleo del polipéptido B2M como marcador para determinar el desarrollo de una enfermedad renal en esta investigación, tuvo fundamentalmente, un juicio económico y de disponibilidad, teniendo en cuenta que nuestro objetivo estaba más centrado en la determinación de la presencia del padecimiento y no en la cualificación de su nivel de progreso. Además de que, con las limitaciones 
volumétricas de la muestra, evaluaciones más profundas no resultarían significativos estadísticamente. Siendo así y en tanto que la primera aplicación clínica de la B2M fue la monitorización de procesos que cursan con disfunción renal, contando con los recursos para realizar el examen a los sujetos y teniendo en cuenta que los resultados de la misma eran factibles a los objetivos del estudio, se prefirió esta prueba por sobre otras que implicarían solo un gasto más elevado y una información más específica, pero sin aportes importantes a los resultados, en el aspecto de la determinación de tendencias por modelación matemática o estadísticas.

Se han descrito numerosos factores de riesgo para el inicio y la progresión de la Enfermedad Renal, cada uno con mayor o menor influencia, según las características de cada estudio. Lo cierto es que la mayoría de estos ha demostrado más asociación que causalidad, la que en muchas ocasiones se presenta de forma inconstante. En cambio, la coexistencia simultánea sí es frecuente, potenciando las repercusiones y el daño. Varios tienen mecanismos fisiopatológicos comunes, siendo la proteinuria y la hiperfiltración glomerular los más frecuentes e importantes.

Entre los más notables se encuentran:

Condiciones no modificables:

Edad, sexo, raza, peso al nacer.

Alteraciones comórbidas potencialmente modificables:

HTA, Diabetes Melitus, obesidad, dislipidemia, tabaquismo, hiperuricemia, hiperalbuminemia, enfermedad cardiovascular.

Alteraciones inherentes a las enfermedades renales:

Anemia, alteraciones del metabolismo mineral, acidosis metabólica. (Lorenzo, 2017)

La B2M es un polipéptido de bajo peso molecular (11.800 D) detectado por primera vez en la orina de pacientes con enfermedad de Wilson y envenenamiento por cadmio en 1968 por Berggard y Bearn. Es identificada como la cadena ligera constante del antígeno del locus antigénico de histocompatibilidad humano (HLA), importante en el proceso de reconocimiento celular, aparece en la superficie de las células con núcleo del sistema sanguíneo abundantemente en linfocitos y monocitos y en varias líneas tumorales, excepto los eritrocitos y la capa trofoblástica de la placenta. (Cruz Nina, García R., \& Guzmán, 2010) 
Sintetizada por numerosas células, sus concentraciones séricas dependen, fundamentalmente, de la renovación de la membrana celular (tasa de síntesis o liberación hacia la reserva sérica) y de la velocidad de aclarado, ya que se elimina por filtración glomerular, siendo posteriormente reabsorbida y catabolizada ${ }^{1}$ por los túbulos renales proximales, haciendo que su determinación cuantitativa en la orina, suero o plasma pueda servir como factor de pronóstico en el tratamiento de diversas patologías. Habitualmente su cuantificación se realiza mediante enzimoinmunoanálisis de micropartículas, turbidimetría o nefelometría. (Gazapo E. G., 1996)

Su nivel en suero aumenta como consecuencia de los problemas de reabsorción glomerular y activación linfocitaria, en ciertos pacientes que sufren patologías inflamatorias autoinmunes (artritis reumatoide, lupus eritematoso sistémico, sarcoidosis), infecciones virales (virus de Epstein Barr, citomegalovirus, influencia A, HIV) y algunos tipos de cáncer como leucemia, linfoma maligno, mieloma, neoplasias de mama, pulmón o estómago. Respecto a la orina, solo pequeñas cantidades se detectan en condiciones normales ya que el $99,9 \%$ de la Beta2- Microglobulina que pasa a través de los glomérulos se reabsorben en los túbulos proximales renales. Aumenta significativamente en las defunciones tubulares renales o cuando los niveles en suero son superiores al nivel de reabsorción tubular (usualmente $>4 \mathrm{mg} / \mathrm{mL}$ ).

La Turbidimetría es una técnica que se sustenta básicamente en los fenómenos ópticos que ocurren durante el paso de un haz de luz a través de un medio. La implementación de técnicas de medición cada vez más novedosas ha permitido realizar mediciones de turbidez que incluyen una buena linealidad y estabilidad, amplios intervalos y la posibilidad de determinar concentraciones específicas de la muestra, incluso en presencia de colorantes. La introducción de nuevos sistemas ópticos para medir indicadores a nivel celular en los procesos biológicos continúa creciendo, constituyéndose en una estrategia efectiva de control. De igual manera, el continuo desarrollo de sensores ofrece cada vez mejores posibilidades de medición

\footnotetext{
${ }^{1}$ El término catabolismo es un término que se utiliza en la biología y en la medicina para hacer referencia al proceso orgánico mediante el cual se reducen diferentes elementos a sus formas más simples, a las moléculas que los compusieron en un momento antes de volverse complejas.
} 
sin perturbaciones y con una mayor precisión en los resultados. (Acebo González \& Hernández García, 2013)

La investigación pretendió comprobar la hipótesis de que "más del 15\% de los miembros de la asociación presenta índices altos de B2M y por tanto desarrollan una enfermedad renal".

\section{METODOLOGÍA}

Se emplearon métodos analítico - descriptivos de corte trasversal. La selección de los sujetos se realizó a partir de un muestreo no probabilístico, con un carácter cualitativo, del tipo muestras por conveniencia que acaparó a 49 individuos de la Asociación del Adulto Mayor en el cantón Jipijapa, quienes fueron sometidos bajo su consentimiento expreso, a exámenes de plasma sanguíneo para determinar la concentración de Beta2-Microglobulina por turbidimetría, encuestados para establecer en sus hábitos y conductas el efecto de los factores de riesgo para el desarrollo de la enfermedad renal y a quienes se revisó su historia clínica para excluir los que padecieran de otras condiciones médicas anteriores que influyeran en el incremento de la concentración del analito como cáncer, linfomas, infecciones virales o patologías inflamatorias.

La concentración de B2M fue considerada variable independiente y la enfermedad renal como variable dependiente. Se emplearon como técnicas los exámenes practicados, las encuestas realizadas a los participantes y la revisión documental de las historias clínicas, con los instrumentos: ficha de registro, cuestionarios, bases de datos y los materiales, equipos y utensilios de laboratorio descritos en los anexos.

Una vez realizados los exámenes y aplicadas las encuestas, se acopiaron los datos obtenidos en la ficha de registro y se revisaron las historias clínicas para descartar otras influencias en la concentración de B2M. Compilados los datos, se procesó la información con el software estadístico SPSS de IBM, segmentando los grupos de edades o géneros y determinando la frecuencia para establecer las comparaciones y análisis pertinentes. 


\section{RESULTADOS Y DISCUSIÓN}

Los exámenes arrojaron que, el 20,8\% del género masculino (5 individuos) mantenían valores por encima de lo normal, así como el $28 \%$ de las féminas (12 mujeres), por lo que del total de la muestra fueron identificados con valores superiores al permisible el $24,5 \%$ de los ancianos. Ver Tabla No 1.

En cuanto a la identificación de los factores de riesgo asociados a la enfermedad renal se pudo determinar, según las encuestas, en el caso de los no modificables, que además de la edad, el resto (sexo, raza y peso al nacer) no fueron significativos, ni representan una influencia notable, salvo que el género femenino reflejó tener un ligero incremento sobre el masculino, en contraposición con lo que refiere la literatura consultada.

Según Lorenzo Sellarés, en Enfermedad Renal Crónica, publicado en la revista Nefrología digital (2017), aunque la edad avanzado es uno de los factores de riesgo más conocidos para la ERC, no constituye un elemento de progresión en sí mismo, más allá del deterioro funcional natural del riñón asociado con la edad, y hasta en algunos estudios aparece paradójicamente como un factor de protección, aludiendo que los pacientes añosos que llegan a las consultas son menos proteinúricos. Se asume, de acuerdo con los cambios de la hemodinámica renal con el envejecimiento,

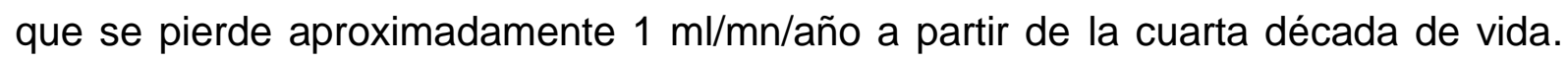
También que, aunque con el envejecimiento se constata esclerosis glomerular, atrofia tubular, y esclerosis vascular, en algunos individuos no se observa el descenso esperado del filtrado.

Refiere el autor que el sexo masculino, es el más proclive en estudios poblacionales, como factor pronóstico independiente de padecer ERC, pero que esta tendencia no ha sido totalmente verificada existiendo varias circunstancias asociados al sexo que son determinantes en la evolución del daño renal. Aun así, en la mayoría de las investigaciones con registros de enfermos renales, los hombres representan aproximadamente el $60 \%$ de los pacientes en tratamiento sustitutivo.

En los factores potencialmente modificables, predominó el consumo frecuente de fármacos, aunque estos no conferían efectos nocivos directos al riñón. Predominando la medicación para el tratamiento de la Hipertensión Arterial (HTA) y la Diabetes 
Mellitus tipo2, dolencias que presentaron entre ambas, el $47 \%$ de los encuestados. Por lo demás, ninguno consume sustancias tóxicas, ni se constatan signos de obesidad en los individuos, tampoco se recogen evidencias de dislipemia, hiperuricemia o hiperalbuminemia. Ver Tabla No 2.

El incremento de la esperanza de vida incide también en el índice de enfermedades crónico degenerativas, razón por la cual, el adulto mayor presenta comúnmente más de una patología que lo obliga a la imposición de esquemas de tratamiento con más de un fármaco llegando en no pocas ocasiones a la polifarmacia. $Y$ esta tendencia representa una amenaza para la salud, pues trae consigo el riesgo de incumplimiento de tratamientos, diminución de la eficacia clínica, interacciones medicamentosas y mayor número de efectos adversos. Todo lo cual se vuelve más frecuente y peligroso por los cambios farmacocinéticos y farmadinamicos que se producen con la edad.

De los inherentes a la enfermedad renal crónica, solo el 3\% reportaba anemia y ninguno presentaba alteraciones en la homeostasis del Calcio $(\mathrm{Ca})$ o el Fósforo $(\mathrm{P}) \mathrm{O}$ trastornos en la hormona polipeptídica (PHT) o indicios de acidez metabólica.

Los registros antes expuestos confirman la prevalencia de HTA y Diabetes en la población mayor de 40 años en el país, informada por el Ministerio de Salud Pública (MSP) y descritas en las cifras del Instituto Nacional de Estadísticas y Censos (INEC). La relación entre los factores de riesgo a que están expuestos los ancianos que participaron del estudio y los niveles de concentración de beta2-microglobulina se hace evidente por cuanto todos los individuos que presentaron algunas o varias de las afecciones analizadas coincidieron con cuotas por encima de lo normal del polipéptido, haciendo evidente su tránsito hacia el desarrollo de una enfermedad renal. En este caso particular, no se comprueban afectaciones de otras circunstancias que favorecieran las probabilidades de contraer o desarrollar la dolencia, como exámenes para la determinación de cáncer o linfomas. Hubiera resultado interesante establecer la proporción del crecimiento de la concentración del polipéptido según las afectaciones encontradas, pero dada la magnitud reducida de la muestra, no aportaría cifras objetivamente referenciales. Ver Tabla No 3.

Como información complementaria se pudo establecer lo siguiente: 
- La mayoría de los encuestados (73\%) alude no tener referencias o conocimiento de los riesgos y/o proceso de la enfermedad.

- El $86 \%$ de los ancianos afirma la ausencia de antecedentes familiares con el padecimiento, por lo que consideramos que este elemento no parece tener una influencia determinante para su desarrollo.

- Referente a las sintomatologías, un $51 \%$ de los adultos mayores presentan dolor lumbar siendo uno de los síntomas con mayor prevalencia, en un 19\% presenta cansancio y fatiga, un $14 \%$ ardor al orinar, el 10\% retención de líquidos en la orina, y por ultimo un $6 \%$ presenta náuseas y vómito.

- Los fármacos que más utilizan se corresponden con las enfermedades preponderantes, de allí que el $41 \%$ no consuma medicación regularmente, mientras el $29 \%$ toma Losartan, el 14\% Metformina, el $12 \%$ Ibuprofeno y un $4 \%$ Aspirina, estos últimos regularmente,

Concluidos los análisis correspondientes, se corrobora la hipótesis sugerida, en vistas a que el $24,5 \%$ de los miembros de la asociación y adultos mayores presentaron niveles de B2M por encima de lo normal. Se tuvo en cuenta, además, para esta afirmación, que el resultado de las encuestas, así como la revisión de las historias clínicas y chequeos de salud realizados con anterioridad y consultados al efecto, no evidenciaron condiciones (patologías inflamatorias, infecciones virales, linfomas o cáncer del pulmón, pecho o estómago) que propiciaran el incremento del analito.

\section{CONCLUSIONES Y RECOMENDACIONES}

Culminado el estudio se comprobó que, a pesar de tener hábitos de vida bastante saludables, sin prácticas tóxicas, índices de masa corporal adecuados a sus parámetros físicos y un nivel de actividad poco sedentario, las afectaciones a la salud más comunes en la región, en conjunto con el proceso normal de deterioro de órganos y funciones por envejecimiento, ha incidido en que un $24,5 \%$ de los ancianos sometidos al estudio presenten concentraciones de Beta2-Microglobulina por encima de los parámetros aceptados, por lo que se estima que muestren enfermedad renal en algún grado. 
Una vez confirmado que las afectaciones de la Hipertensión Arterial y la Diabetes Mellitus constituyen los principales factores que terminan derivando en una enfermedad renal, es importante actuar sobre los canales y mecanismos de información de los actores de salud en la región, para que a través de la divulgación sobre el conocimiento de sus causas y efectos sobre la calidad de vida, se logren transformar las costumbres nocivas con vistas a minimizar los causales modificables de estas enfermedades.

Siendo la población de adultos mayores un grupo de alta vulnerabilidad, sería recomendable establecer programas que incentiven los chequeos periódicos de la tensión arterial y los niveles de glucosa, con el objetivo de mantenerlos controlados y detectar de manera precoz el desarrollo de enfermedades que aceleran el deterioro de sistemas y órganos, afectando la salud y la calidad de vida de este sector tan importante de la sociedad.

\section{ANEXOS}

Tabla No.1 GÉNERO *Valores normales y altos tabulación cruzada

\begin{tabular}{llccc}
\hline & & \multicolumn{3}{c}{ Valores } \\
& & alto & normal & Total \\
\hline GENERO Femenino & Recuento & 7 & 18 & 25 \\
& \% dentro de GÉNERO & $28.0 \%$ & $72.0 \%$ & $100.0 \%$ \\
\multirow{4}{*}{ Total } & Recuento & 5 & 19 & 24 \\
& \% dentro de GÉNERO & $20.8 \%$ & $79.2 \%$ & $100.0 \%$ \\
& Recuento & 12 & 37 & 49 \\
& \% dentro de GÉNERO & $24.5 \%$ & $75.5 \%$ & $100.0 \%$ \\
\hline
\end{tabular}

Fuente: Encuestas en la Asociación del Adulto Mayor del Cantón Jipijapa 


\begin{tabular}{|c|c|c|c|c|c|c|}
\hline $\begin{array}{rr}\text { Tabla No.2 Ries } \\
\text { Tabla No.3 Enfer } \\
\text { de B2 }\end{array}$ & $\begin{array}{l}\text { difi } \\
\text { es a }\end{array}$ & $\begin{array}{l}\text { s vs G } \\
\text { das vs }\end{array}$ & os & $\begin{array}{l}\text { des } \\
\text { n alt }\end{array}$ & & ciones \\
\hline & FEI & & $\mathrm{M}$ & INO & & \\
\hline Alternauva & $f$ & $\%$ & $f$ & $\%$ & $f$ & $\%$ \\
\hline Diabetes & 3 & 25.0 & 2 & 16.7 & 4 & 41.7 \\
\hline Hipertensión & 4 & 33.3 & 3 & 25.0 & 7 & 58.3 \\
\hline Anemia & 0 & 0.0 & 1 & 8.3 & 1 & 8.3 \\
\hline
\end{tabular}

Fuente: Encuestas en la Asociación del Adulto Mayor del Cantón Jipijapa

\section{DIAGNÓSTICO DE LABORATORIO}

Técnica de la prueba: Beta 2 - Microglobulina Turbidimetría.

\section{Fundamento}

Las partículas de látex sensibilizadas con anti-B2-microglobulin humana, son aglutinadas cuando reaccionan con la B2M presente en la muestra. La aglutinación de las partículas de látex es proporcional a la concentración de B2M en la muestra y puede ser medida por turbidimetría.

\section{Composición de reactivos}

Reactivo 1: Diluente. Tampón tris, $20 \mathrm{mmol} / \mathrm{L}, \mathrm{pH} 8,2$

Reactivo 2: Látex suspensión de partículas de látex sensibilidad con igG de cabra anti(B2-M) humana, $\mathrm{pH}$ 8,2.

CAL-S Calibrador de suero. Suero humano.

CAL-U Calibrador de orina. Orina humana.

La concentración de beta - microglobulina viene indicada en la etiqueta del vial y es trazable al $1^{\circ}$ estándar para Beta 2- microglobulina, 


\section{Precauciones}

Los reactivos del kit contienen azida sódica $\left(\mathrm{NaN}_{3}\right)$ 0,95 g/L. evitar el contacto con la piel y mucosas.

Los componentes del kit de origen humano han dado resultados negativos frente a anticuerpos de HIV, HBsAg y anti-HCV. Sin embargo, se recomienda tomar precauciones durante su uso.

\section{Preparación de los reactivos}

R1. Listo para su uso.

R2. Listo para su uso. Mezclar suavemente por inversión del vial antes de usar

CAL. Listo para su uso.

\section{Almacenamiento y estabilidad}

1. Todos los reactivos son estables hasta la fecha de caducidad indicada en la etiqueta del vial, cuando se mantiene bien cerrados a $2-8^{\circ}$ y se evita la contaminación durante su uso. No usar los reactivos una vez caducados.

2. Indicadores de deterioro de los reactivos: presencia de partículas y turbidez.

\section{Muestras}

Suero fresco. Estable de 7 días a $2-8^{\circ} \mathrm{C}$ o 3 meses a $-20^{\circ} \mathrm{C}$. Centrifugar las muestras con restos de fibrina antes de usar. No utilizar muestras altamente hemolizada o lipémicas.

Orina fresca. Ajustar el pH a 7,6 con una solución de 0,4 mol/L de $\mathrm{K}_{2} \mathrm{HPO}_{4}$. Estable 2 días a $2-8^{\circ} \mathrm{C}$ o 2 meses a $-20^{\circ} \mathrm{C}$.

\section{Equipo adicional}

- Baño de agua a $37^{\circ} \mathrm{C}$.

- Espectrofotómetro o fotómetro con cubeta termostatizable a $37^{\circ} \mathrm{C}$ para lecturas a $540 \pm 20 \mathrm{~nm}$.

\section{Técnica}

Procedimiento preliminar 
Precalentar los reactivos y el fotómetro (portacubetas) a $37^{\circ} \mathrm{C}$

Procedimiento analítico

1. Ajustar el cero del instrumento a $540 \mathrm{~nm}$ con agua destilada.

2. Pipetear en una cubeta.

\begin{tabular}{l|ll} 
& Suero & Orina \\
\hline $\begin{array}{l}\text { Diluente(R1) } \\
\text { Muestra, }\end{array}$ & $0,8 \mathrm{ml}$ & $0,8 \mathrm{ml}$ \\
$\begin{array}{l}\text { CAL-S, } \\
\text { CAL-U }\end{array}$ & $10 \mu \mathrm{l}$ & $50 \mu \mathrm{l}$ \\
Látex (R2) & $0,2 \mathrm{ml}$ & $0,2 \mathrm{ml}$
\end{tabular}

3. Mezclar e insertar la cubeta en el fotómetro. Leer la absorción inmediatamente $\left(A_{1}\right)$ y a los 3 minutos $\left(A_{2}\right)$ de la adición de la muestra.

\section{Cálculos}

$$
\text { B2M }(\mathrm{mg} / \mathrm{l})=\frac{\left(A_{2}-A_{1}\right) \text { muestra }}{\left(A_{2}-A_{1}\right) \text { calibrador }} * \text { CAL conc }
$$

\section{Control De Calidad}

Se recomienda utilizar sueros control, plasma, proteína control N- I y N-Il para controlar los ensayos tanto en procedimiento manual como en automático.

Cada laboratorio debería establecer su propio Control de Calidad y establecer correcciones en el caso de que los controles no cumplan con las tolerancias exigidas.

\section{Valores De Referencia}

Suero: $1-3 \mathrm{mg} / \mathrm{l}$

Orina: $<0,3 \mathrm{mg} / \mathrm{l}$

Es recomendable que cada laboratorio establezca sus propios valores de referencia.

\section{Significado clínico}

La Beta 2- Microglobulina es un polipéptido de bajo peso molecular. Es un componente de complejo de histocompatibilidad. Los linfocitos producen el $50 \%$ de la Beta 2- 
Microglobulina que se filtra libremente a través de la membrana basal glomerular. Por lo que el nivel de beta 2-microglobulina en suero aumenta como consecuencia de los problemas de reabsorción glomerular y activación linfocitaria. Diversos estudios muestran que se encuentran niveles elevados de Beta 2- microglobulina en suero de ciertos pacientes que sufren patologías inflamatorias (artritis reumatoide, lupus eritematoso sistémico, sarcoidosis), infecciones virales (virus de Epstein Barr, citomegalovirus, influencia A, HIV), linfomas (leucemia, linfoma maligno, mieloma) y cáncer (pecho pulmón, estómago)

Respecto de la Beta 2- Microglobulina en orina, solo pequeñas cantidades se detectan en condiciones normales ya que el $99,9 \%$ de la beta 2- microglobulina que pasa a través de los glomérulos se reabsorben en los túbulos proximales renales. Además, el nivel de Beta 2- Microglobulina aumenta significativamente en las defunciones tubulares renales o cuando los niveles en suero son superiores al nivel de reabsorción tubular (usualmente $>4 \mathrm{mg} / \mathrm{ml}$ ).

\section{Características analíticas}

Límite de linealidad.

Hasta los $18 \mathrm{mg} / \mathrm{l}$ (suero), $3 \mathrm{mg} / \mathrm{l}$ (orina) en las condiciones descritas del ensayo. Las muestras con valores superiores deben diluirse $1 / 5$ en $\mathrm{CINa} 9 \mathrm{~g} / \mathrm{L}$ y repetirse el ensayo.

\section{Límite de detección.}

Valores por debajo de $0,15 \mathrm{mg} / \mathrm{l}$ dan lugar a resultados poco reproducibles.

Sensibilidad analítica: $30 \mathrm{~mA} / \mathrm{mg}$ (B2M) //.

Efecto prozona: No se observan hasta valores de $100 \mathrm{mg} / \mathrm{L}$.

Exactitud: Los resultados obtenidos con estos reactivos no muestran diferencias significativas cuando se comparan con reactivos de características similares.

\section{Interferencias}

Suero: La bilirrubina $(40 \mathrm{mg} / \mathrm{dl})$, la hemoglobina $(12 \mathrm{~g} / \mathrm{l})$, la lipemia $(>20 \mathrm{~g} / \mathrm{l})$, y los factores reumatoides $(300 \mathrm{ul} / \mathrm{ml})$, no interfieren.

Orina: Hemoglobina $(12 \mathrm{~g} / \mathrm{l})$, la creatinina $(300 \mathrm{mg} / \mathrm{l})$, el ácido úrico $(500 \mathrm{mg} / \mathrm{l})$ y la urea $(100 \mathrm{mg} / \mathrm{l})$, no interfieren. 


\section{REFERENCIAS CONSULTADAS}

1. (s.f.). Recuperado el Julio de 2018, de Ministerio de Salud Pública Ecuador website:

https://aplicaciones.msp.gob.ec/salud/archivosdigitales/sigobito/tareas segui miento/1469/Presentaci\%C3\%B3n\%20Di\%C3\%A1lisis\%20Criterios\%20de\%2 0Priorizaci\%C3\%B3n\%20y\%20Planificaci\%C3\%B3n.pdf

2. Acebo González, D., \& Hernández García, A. (enero-abri de 2013). Los métodos turbidimétricos y sus aplicaciones en las ciencias de la vida. Revista CENIC. Ciencias Biológicas, 44(1).

3. Castro Jalca, A., \& Marcillo Proaño, E. (2017). Beta-2 Microglobulina para detección de enfermedad renal en la Asociación del Adulto Mayor del cantón Jipijapa. Tesis de Grado, Universidad Estatal del Sur de Manabí (UNESUM), Facultad de Ciencias de la Salud, Carrera Laboratorio Clínico, Jipijapa.

4. Cruz Nina, S., García R., H., \& Guzmán, M. (Junio de 2010). Caracterización de resultados obtenidos de beta2-microglobulina en orina de pacientes diagnosticados con diabetes tipo 2 con métodos de rutina clínica aplicables a esta patología. Biofarbo, Revistas Bolivianas, 18(1). Obtenido de http://www.revistasbolivianas.org.bo/scielo.php?pid=S1813-

$\underline{53632010000100005 \& \text { script }=\text { sci arttext }}$

5. Espinosa Sevilla, A., Amezcua Macías, A., Ruiz Palacios, P., Rodríguez Weber, F., \& Díaz Greene, E. (2013). Nuevos marcadores de lesión renal aguda en el enfermo grave. Medicina Interna México, 29(513-519).

6. Fernández Vega, F., Marín, R., Riesgo, A., Herrero, P., Prieto, M., Trance, S., \& et.al. (2005). Recuperado el Julio de 2018, de Revista Nefrología web site: http://revistanefrologia.com/es-publicacion-nefrologia-articulo-insuficienciarenal-cronica-progresion-irc-X0211699505031161

7. Gazapo, E. G. (Mayo de 1996). Utilidad clínica de la determinación de beta-2microglobulina. Rev. Med Clin (Barc), 106, 751-5.

8. Gazapo, E., Gazapo, R., \& Caturla, A. (1996). Utilidad clínica de la determinación de bata-2-microglobulina (Vol. 106). Barcelona, España: Abbot Científica. Recuperado el Julio de 2018, de http://files.sld.cu/medicinainterna/files/2009/07/beta-2-microglobulina.pdf

9. Lorenzo, V. (2017). Obtenido de Nefrología Digital: http://revistanefrologia.com/es-monografias-nefrologia-dia-articuloenfermedad-renal-cronica-136 
10. Organización Mundial de la Salud (OMS). (2018). Recuperado el Julio de 2018, de Organización Panamericana de la Salud web site: https://www.paho.org/hq/index.php?option=com content\&view=article\&id=141 16\%3Awebinar-kidneys-womens-health-8-march2018\&catid $=7261 \% 3$ Aevents -1 \&ltemid $=41080$ \&lang $=e s$

\section{REFERENCES CONSULTED}

1. (s.f.). Retrieved on July 2018, from the Ministry of Public Health Ecuador website:

https://aplicaciones.msp.gob.ec/salud/archivosdigitales/sigobito/tareas segui miento/1469/Presentaci\%C3\%B3n\%20Di\%C3\%A1lisis\%20Criterios\%20of\%2 OPriority\%C3\%B3n\%20y\%20Planning\%C3\%B3n.pdf

2. Acebo González, D., \& Hernández García, A. (January-April 2013). The turbidimetric methods and their applications in the life sciences. CENIC Magazine. Biological Sciences, 44 (1).

3. Castro Jalca, A., \& Marcillo Proaño, E. (2017). Beta-2 Microglobulin for detection of kidney disease in the Association of the Elderly of the Jipijapa canton. Undergraduate thesis, State University of Southern Manabí (UNESUM), Faculty of Health Sciences, Clinical Laboratory Career, Jipijapa.

4. Cruz Nina, S., García R., H., \& Guzmán, M. (June 2010). Characterization of results obtained from beta2-microglobulin in urine of patients diagnosed with type 2 diabetes with routine clinical methods applicable to this pathology. Biofarbo, Bolivian Magazines, 18 (1). Retrieved from http://www.revistasbolivianas.org.bo/scielo.php?pid=S1813$\underline{53632010000100005 \& \text { script }=\text { sci arttext }}$

5. Espinosa Sevilla, A., Amezcua Macías, A., Ruiz Palacios, P., Rodríguez Weber, F., \& Díaz Greene, E. (2013). New markers of acute kidney injury in the seriously ill. Internal Medicine Mexico, 29 (513-519).

6. Fernández Vega, F., Marín, R., Riesgo, A., Herrero, P., Prieto, M., Trance, S., \& et.al. (2005). Recovered on July 2018, from Revista Nefrología web site: http://revistanefrologia.com/es-publicacion-nefrologia-articulo-insuficienciarenal-cronica-progresion-irc-X0211699505031161

7. Gazapo, E. G. (May 1996). Clinical utility of the determination of beta-2microglobulin. Rev. Med Clin (Barc), 106, 751-5.

8. Gazapo, E., Gazapo, R., \& Caturla, A. (1996). Clinical utility of the determination of bata-2-microglobulin (Vol. 106). Barcelona, Spain: Abbot Scientific. Retrieved 
on July 2018, from http://files.sld.cu/medicinainterna/files/2009/07/beta-2$\underline{\text { microglobulina.pdf }}$

9. Lorenzo, V. (2017). Obtained from Digital Nephrology: http://revistanefrologia.com/es-monografias-nefrologia-dia-articuloenfermedad-renal-cronica-136

10. World Health Organization (WHO). (2018). Retrieved July 2018, from the Pan American Health Organization web site: https://www.paho.org/hq/index.php?option=com content\&view=article\&id=141 16\%3Awebinar-kidneys-womens-health-8-march2018\&catid $=7261 \% 3$ Aevents -1 \&ltemid=41080\&lang=en

C2019 por los autores. Este artículo es de acceso abierto y distribuido según los términos y condiciones de la licencia Creative Commons Atribución-NoComercial-Compartirlgual 4.0 Internacional (CC BY-NC-SA 4.0) (https://creativecommons.org/licenses/by-nc-sa/4.0/). 